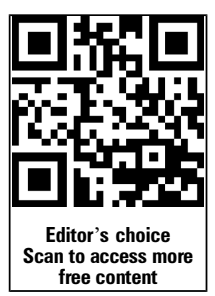

Scan to access $m$ free content
- Additional material is published online only. To view please visit the journal online (http://dx.doi.org/10.1136/ thoraxjnl-2014-206438).

${ }^{1}$ Division of Epidemiology, Department of Chronic Diseases, Norwegian Institute of Public Health, Oslo, Norway ${ }^{2}$ Norwegian Institute of Public Health, Institute Management and Staff, Oslo, Norway ${ }^{3}$ Division of Epidemiology, Department of

Pharmacoepidemiology, Norwegian Institute of Public Health, Oslo, Norway ${ }^{4}$ Medical Faculty, Department of Community Medicine, University of Oslo, Oslo, Norway

${ }^{5}$ Epidemiology Branch, National Institutes of Health National Institute of Environmental Health Sciences, Research Triangle Park, North Carolina, USA

\section{Correspondence to}

Maria Christine Magnus, Norwegian Institute of Public Health, Division of Epidemiology, Department of Chronic Diseases, PO Box 4404, Nydalen, N-0403 Oslo, Norway; maria.christine. magnus@fhi.no

Received 13 October 2014 Revised 5 December 2014 Accepted 12 December 2014 Published Online First

8 January 2015

\section{SLinked}

- http://dx.doi.org/10.1136/ thoraxjnl-2014-206643

\section{CrossMark}

To cite: Magnus MC, Håberg SE, Karlstad Ø, et al. Thorax 2015;70:237-243.

\title{
Grandmother's smoking when pregnant with the mother and asthma in the grandchild: the Norwegian Mother and Child Cohort Study
}

\author{
Maria C Magnus, ${ }^{1}$ Siri E Håberg, ${ }^{2}$ Øystein Karlstad ${ }^{3}$ Per Nafstad, 1,4 \\ Stephanie J London, ${ }^{5}$ Wenche Nystad ${ }^{1}$
}

\section{ABSTRACT}

Background A trans-generational influence of prenatal tobacco smoke exposure on asthma development has been proposed but the evidence remains sparse. Methods We examined the grandmother's smoking when pregnant with the mother in relation to asthma outcomes in the grandchild (current asthma at 36 months ( $N=53169$, cases=3013), current asthma at 7 years $(N=25394$, cases $=1265)$ and dispensed asthma medications at 7 years in the Norwegian Prescription Database ( $N=45607$, cases $=1787)$ ) within the Norwegian Mother and Child Cohort Study (MoBa). We calculated adjusted RR (adj. RR) and 95\% Cls using log binomial regression.

Results A total of $23.5 \%$ of mothers reported that their mother smoked when pregnant with them. The grandmother's smoking when pregnant with the mother was positively associated with asthma at 36 months (adj. RR $1.15(95 \% \mathrm{Cl} 1.06$ to 1.24)), asthma at 7 years (adj. RR $1.21(95 \% \mathrm{Cl} 1.07$ to 1.37)) and dispensed asthma medications at 7 years (adj. RR $1.15(95 \% \mathrm{Cl}$ 1.04 to 1.26)). This positive association did not differ significantly by the mother's smoking status when pregnant with the child ( $p$ values for multiplicative interaction $>0.1$ ).

Conclusions The grandmother's smoking when pregnant with the mother increased the risk of asthma in the grandchild independent of the mother's smoking status. However, given limited information on the grandmother's socioeconomic status, asthma status and other factors, unmeasured confounding may be present.

\section{INTRODUCTION}

Observational studies indicate that parental smoking is positively associated with asthma development in the child. Meta-analyses summarising the evidence find that the most consistent positive association is seen for maternal smoking during pregnancy. ${ }^{1-3}$ In concordance with these findings, exposure to maternal smoking during pregnancy is associated with decreased lung function both in childhood and adulthood. ${ }^{4-7}$

Whether there are trans-generational effects of tobacco smoke exposure is an interesting question. Trans-generational inheritance of an asthma phenotype from in utero nicotine exposure has been reported in a rat model. ${ }^{8}$ Of the two human studies that have examined whether the grandmother's smoking when pregnant with the mother influenced

\section{Key messages}

What is the key question?

- Does the grandmother's smoking when pregnant with the mother influence asthma development in the grandchild?

What is the bottom line?

- The grandmother's smoking when pregnant with the mother increased the risk of asthma in the grandchild independent of the mother's smoking status.

\section{Why read on?}

- Our findings support the idea that prenatal tobacco smoke exposure might have a trans-generational influence on asthma development.

asthma in the grandchild, one found a positive association while the other reported no association. ${ }^{9} 10$ A biological plausibility exists for a transgenerational influence of prenatal tobacco smoke exposure on asthma development. Three previous studies reported that the grandmother's smoking when pregnant with the mother might increase the grandchild's birth weight. ${ }^{11-13}$ Birth weight is also a predictor for asthma. ${ }^{14}{ }^{15}$ Differential methylation, an epigenetic alteration, has also been reproducibly observed in newborns whose mothers smoked during pregnancy. ${ }^{16}$ Epigenetic mechanisms therefore constitute one underlying mechanisms which might explain a trans-generational effect of tobacco smoke exposure.

Given the limited human data on potential transgenerational effects of prenatal tobacco smoke exposure with asthma development, we examined the association between the grandmother's smoking when pregnant with the mother and asthma development in the grandchild using the Norwegian Mother and Child Cohort Study (MoBa).

\section{METHODS}

\section{Study population}

$\mathrm{MoBa}$ is a prospective population-based pregnancy cohort. ${ }^{17} 18 \mathrm{MoBa}$ recruited pregnant women between 1999 and 2008, at approximately 
18 weeks' gestation. The participation rate was $40.6 \%$ of all invited pregnant women. Mothers could participate with more than one pregnancy, resulting in approximately 95200 mothers and 114500 children. All participants gave written informed consent. In May 2014, the 114761 children participating in MoBa were linked to the Medical Birth Registry of Norway (henceforth referred to as 'birth registry') and the Norwegian Prescription Database (henceforth referred to as 'prescription registry') using unique personal identification numbers. Children not linked to the birth registry $(n=499)$ and children from multiple births $(n=3971)$ were not eligible for the current study. We used information from questionnaires completed at 18 and 30 gestational weeks, and when the child was 6 months, 36 months and 7 years. ${ }^{19}$ The Norwegian Data Inspectorate and the Regional Ethics Committee for South/East Norway approved this study. The study is reported according to the Strengthening the Reporting of Observational Studies in Epidemiology (STROBE) recommendations.

\section{Exposure}

Mothers in MoBa were asked 'did your mother smoke when she was pregnant with you?' at approximately 18 gestational weeks. The response alternatives were 'yes', 'no' and 'don't know'. This information identified the grandmother's smoking when she was pregnant with the mother. Mothers further reported their own smoking habits during pregnancy at 18 gestational weeks, 30 gestational weeks and retrospectively when the child was 6 months. To evaluate whether the grandmother's smoking had an independent association with asthma development in the grandchild from maternal smoking during pregnancy, we classified the children into four mutually exclusive exposure groups: not exposed to smoking, only exposed to the grandmother's smoking, only exposed to the mother's smoking during pregnancy, and both the grandmother and the mother smoked.

\section{Outcome}

We examined two asthma outcomes based on the MoBa questionnaires and one outcome based on asthma medications as registered in the prescription registry. (1) We defined current asthma at 36 months as a 'yes' by the mother to a question about current asthma in combination with reported names of inhaled glucocorticoids and/or beta(2) agonists in an openended question of which medications the child had used during the past year. (2) Current asthma at 7 years was defined based on the mother's report that the child had experienced asthma symptoms in the past year in combination with 'yes' to a closed-ended question of whether the child had used any medications for asthma in the past year. (3) We classified a child as having redeemed asthma medications based on the prescription registry if there was at least one dispensed prescription for asthma medications in the past year when the child was 7 years in addition to a second dispensed prescription within 1 year of the first. The prescription registry classifies medications according to the Anatomical Therapeutic Chemical classification system. Medications classified as asthma medications included inhaled short-acting and long-acting $\beta(2)$ agonists (R03AC), inhaled corticosteroids (R03BA), fixed-dose combinations of inhaled $\beta(2)$ agonists and corticosteroids (R03AK), and leukotriene antagonists (R03DC).

\section{Covariates/confounders}

Characteristics plausibly associated with the grandmother's smoking and child asthma development were included as potential confounders. Since we did not have information concerning socioeconomic or health characteristics of the grandmother, we relied on maternal characteristics as indirect measures. Maternal age at delivery, parity (primiparous, 1, 2 and 3 or more), education (less than high school, high school, up to 4 years of college and more than 4 years of college), yearly salary $(<200000$, $200000-400000$ and >400000 NOK), and pre-pregnancy body mass index (BMI) (underweight, normal weight, overweight and obese; categorised according to WHO guidelines) were included as indicators of socioeconomic status/health status. Maternal asthma was included as an indicator of genetic predisposition. The child's birth weight was considered as a potential intermediate factor.

\section{Statistical analysis}

We used log binomial regression to estimate RR and 95\% CIs for asthma in the grandchild related to the grandmother's smoking when pregnant with the mother. In the first multivariable regression model we adjusted for all the potential confounding factors specified (model 1), reporting adjusted RR (adj. RR) and 95\% CI. We used robust cluster variance estimations to account for siblings. The second multivariable model adjusted for all factors included in model 1 in addition to maternal smoking during pregnancy (model 2). We tested for multiplicative interaction of the mother's smoking during pregnancy by including product terms in the multivariable regression analysis. Furthermore, the grandmother's and the mother's smoking was evaluated in combination, classified into four mutually exclusive exposure categories. Finally, we examined the influence of adjustment for the potential intermediate factor: the child's birth weight. In the multivariable analysis, up to $10 \%$ of observations had missing information on one or more of the covariates. To evaluate the potential influence of missing covariate information we conducted multiple imputation by chained equations, imputing a total of 20 datasets. As the results using complete case and multiple imputation were similar, we only present the measures of association from the multiple imputation analysis. The results from the complete case analysis are included in online supplementary tables S2 and S3. We used inverse probability weighting to evaluate selection bias. ${ }^{20}$ The weights reflected the probability of having the necessary follow-up information to be included in the respective analysis among all eligible children. The statistical significance level was 5\%. All analyses were conducted in STATAV.13 (Statacorp, Texas, USA).

\section{RESULTS}

Of the 110291 eligible children, 53169 had the follow-up information to be included in the analysis of current asthma at 36 months (figure 1). Among these 53169 children, 41209 had reached 7 years by 1 July 2014, out of which 25394 children had information from the 7 -year questionnaire (figure 1 ). These children were included in the analysis of current asthma at 7 years. A total of 45607 children were included when evaluating children who had dispensed asthma medications at 7 years registered in the prescription registry (figure 1). Online supplementary table S1 indicates the distribution of characteristics within these three study populations.

The grandmother's smoking when pregnant with the mother was reported for $23.5 \%$ of children, while mothers of $66.9 \%$ of children reported that their mother did not smoke when pregnant with them, and for $9.6 \%$ of children the mother reported 'don't know'. Mothers who reported prenatal tobacco smoke exposure were younger, had a higher parity, lower education, lower salary, were more likely to be overweight/obese and more likely to have a history of asthma (table 1). The birth weight of 


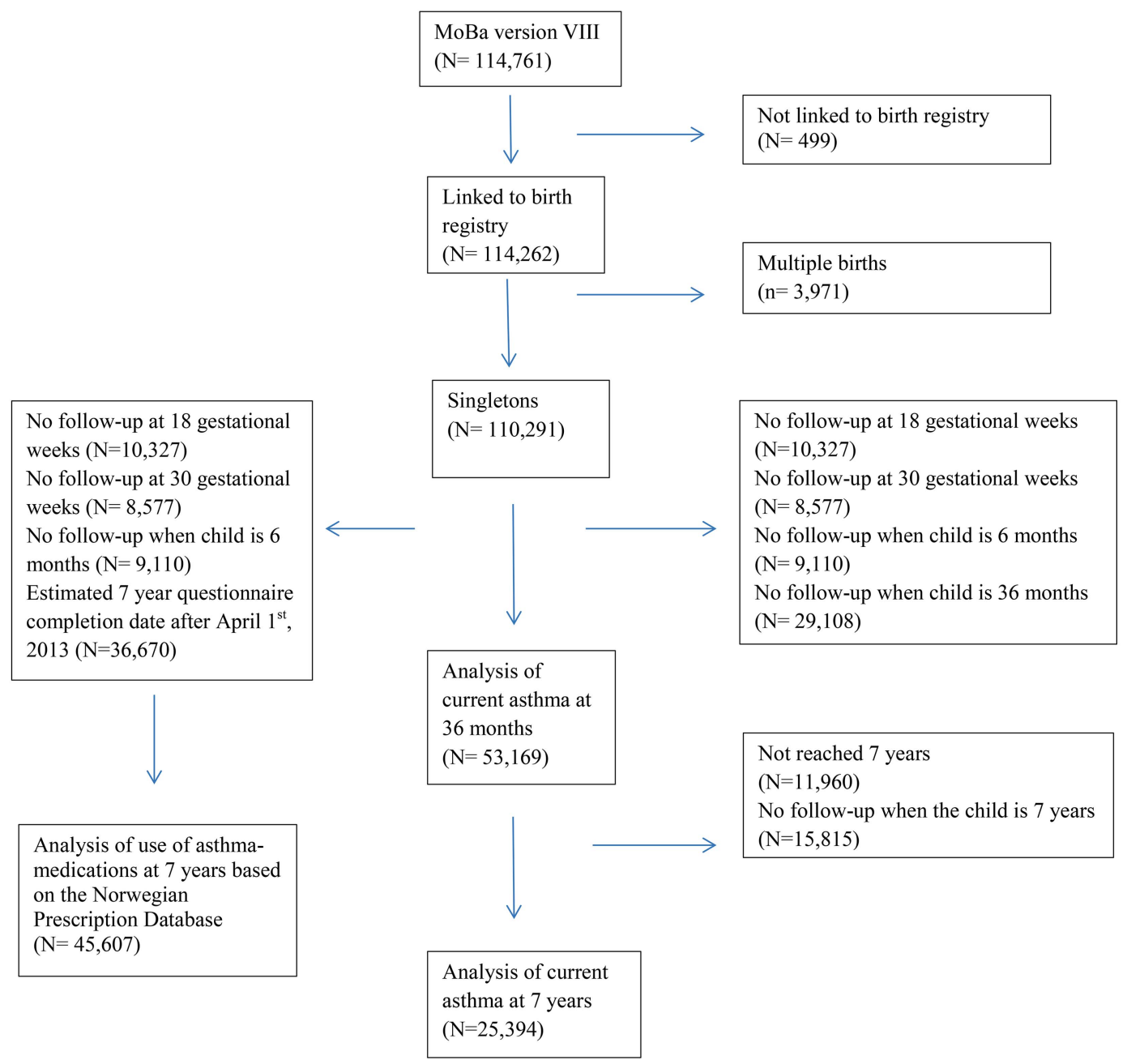

Figure 1 Illustration of sample selection.

the grandchild was also slightly higher if the grandmother had smoked when she was pregnant with the mother (table 1).

Current asthma at 36 months was reported for $5.7 \%$ of children, current asthma at 7 years for $5.1 \%$ while $4.8 \%$ had dispensed asthma medications at 7 years in the prescription registry. The grandmother's smoking when pregnant with the mother was modestly associated with asthma at 36 months, adj. RR 1.15 (95\% CI 1.06 to 1.24 ), asthma at 7 years, adj. RR 1.21 (95\% CI 1.07 to 1.37 ) and dispensed asthma medications in the prescription registry, adj. RR 1.15 (95\% CI 1.04 to 1.26) (model 1, table 2). Maternal age, pre-pregnancy BMI and asthma explained the majority of the change from the crude associations. Further adjustment for maternal smoking during pregnancy did not change the association between the grandmother's smoking and asthma in the grandchild (model 2, table 2). Children of mothers who responded 'don't know' to the question regarding whether their mother smoked when pregnant with them had risk estimates that were positive but smaller than the exposed category for all outcomes (table 2). There was no indication of multiplicative interaction of maternal smoking during pregnancy (all $\mathrm{p}$ values $>0.1$ ).

We also evaluated the grandmother's smoking when pregnant with the mother and maternal smoking when pregnant with the child in combination (table 3). For all three asthma outcomes, exposure to the grandmother's smoking alone, or exposure to the grandmother's smoking and the mother's smoking showed a tendency for a positive association (table 3). Notably, there was no independent statistically significant association of exposure to maternal smoking during pregnancy alone and asthma development.

The birth weight of the grandchild, which has been associated with the grandmother's smoking during pregnancy, could be on the causal pathway to asthma. Further adjustment for the grandchild's birth weight did not change the association between the grandmother's smoking when pregnant with the mother and asthma in the grandchild.

We conducted a number of sensitivity analyses. The mother's asthma status might be considered an intermediate factor. We therefore explored the multivariable analyses with and without adjustment for the mother's asthma status which indicated similar associations (see online supplementary table S4). Further multivariable adjustment for the mother's smoking status in the first 6 months of the child's life did not change the association between the grandmother's smoking and asthma in the grandchild (see online supplementary table S4, model 3). Likewise, multivariable adjustment for the father's asthma status and the father's smoking status during pregnancy and the first 6 months of the child's life also left the association unchanged (see online supplementary table S4, model 4). Furthermore, there was no difference in the associations by the child's gender (all $\mathrm{p}$ values for interaction $>0.1$ ). Finally, the results of the analysis using inverse probability weighting also yielded similar results. 
Table 1 Maternal characteristics and child birth weight by the grandmother's smoking when pregnant with the mother

\begin{tabular}{|c|c|c|c|}
\hline Characteristic & $\begin{array}{l}\text { No } \\
(n=34 \text { 859) }\end{array}$ & $\begin{array}{l}\text { Yes } \\
(n=12253)\end{array}$ & $\begin{array}{l}\text { Don't know } \\
(n=4988)\end{array}$ \\
\hline Maternal age at delivery (mean (SD)) & $30.5(4.4)$ & $30.1(4.5)$ & $30.6(4.5)$ \\
\hline \multicolumn{4}{|l|}{ Maternal parity (\%) } \\
\hline Primiparous & 49.1 & 45.0 & 46.7 \\
\hline 1 & 33.4 & 36.0 & 35.1 \\
\hline 2 & 13.7 & 15.0 & 14.6 \\
\hline 3 or more & 3.8 & 4.1 & 3.6 \\
\hline \multicolumn{4}{|l|}{ Maternal education (\%) } \\
\hline Less than high school & 4.1 & 8.7 & 6.3 \\
\hline High school & 23.9 & 32.9 & 30.2 \\
\hline Up to 4 years of college & 44.4 & 40.4 & 41.4 \\
\hline More than 4 years of college & 27.5 & 18.0 & 22.1 \\
\hline \multicolumn{4}{|l|}{ Maternal yearly salary (\%) } \\
\hline$<200000$ NOK & 10.0 & 10.1 & 9.4 \\
\hline $200000-400000$ NOK & 54.7 & 57.5 & 57.3 \\
\hline$>400000$ NOK & 35.3 & 32.4 & 33.3 \\
\hline \multicolumn{4}{|l|}{ Maternal pre-pregnancy BMI (\%) } \\
\hline Underweight $(<18.5)$ & 3.1 & 2.5 & 2.9 \\
\hline Normal weight (18.5-24.9) & 69.2 & 60.3 & 64.0 \\
\hline Overweight (25-29.9) & 20.2 & 25.0 & 23.2 \\
\hline Obese $(\geq 30)$ & 7.4 & 12.3 & 10.0 \\
\hline \multicolumn{4}{|l|}{ Maternal asthma (\%) } \\
\hline No & 93.4 & 91.5 & 91.9 \\
\hline Yes & 6.6 & 8.5 & 8.1 \\
\hline \multicolumn{4}{|l|}{ Maternal smoking during pregnancy (\%) } \\
\hline No & 95.0 & 86.1 & 91.2 \\
\hline Yes & 5.0 & 13.9 & 8.8 \\
\hline Child birth weight, g (mean (SD)) & 3599 (534) & $3626(555)$ & $3605(540)$ \\
\hline
\end{tabular}

\section{DISCUSSION}

In this large-scale population based study, the grandmother's smoking when pregnant with the mother was positively associated with asthma in the grandchild. This positive association was independent of the mother's smoking status when pregnant with the child.
Our findings support those from the Children's Health Study in California indicating a positive association between the grandmother's smoking when pregnant with the mother and asthma in the grandchild by 5 years of age, adj. OR 2.1 (95\% CI 1.4 to 3.2$).{ }^{9}$ In contrast, the ALSPAC study showed no association between the grandmother's smoking when pregnant

Table 2 Association between the grandmother's smoking when pregnant with the mother and asthma development in the grandchild

\begin{tabular}{|c|c|c|c|c|c|c|}
\hline Outcome & Exposure & $\mathrm{n}$, total & $\mathrm{n}$, cases & Crude RR (95\% CI) & $\begin{array}{l}\text { Model } 1 \\
\text { adj. RR (95\% Cl) }\end{array}$ & $\begin{array}{l}\text { Model } 2 \\
\text { adj. RR }(95 \% \mathrm{Cl})\end{array}$ \\
\hline Asthma at 36 months & \multicolumn{6}{|c|}{ Grandmother smoked during pregnancy } \\
\hline \multirow{3}{*}{$(\mathrm{N}=53169)$} & No & 34460 & 1843 & 1 & 1 & 1 \\
\hline & Yes & 12104 & 803 & 1.24 (1.15 to 1.35$)$ & 1.15 (1.06 to 1.24$)$ & $1.15(1.06$ to 1.25$)$ \\
\hline & Don't know & 4933 & 307 & $1.16(1.03$ to 1.31$)$ & $1.11(0.99$ to 1.24$)$ & $1.11(0.99$ to 1.24$)$ \\
\hline Asthma at 7 years & \multicolumn{6}{|c|}{ Grandmother smoked during pregnancy } \\
\hline \multirow[t]{3}{*}{$(\mathrm{N}=25394)$} & No & 16301 & 759 & 1 & 1 & 1 \\
\hline & Yes & 5756 & 348 & 1.31 (1.15 to 1.48$)$ & 1.21 (1.07 to 1.37$)$ & 1.21 (1.07 to 1.37$)$ \\
\hline & Don't know & 2276 & 129 & 1.21 (1.01 to 1.46$)$ & $1.15(0.96$ to 1.39$)$ & $1.15(0.96$ to 1.39$)$ \\
\hline Use of asthma medications at 7 years & \multicolumn{6}{|c|}{ Grandmother smoked during pregnancy } \\
\hline \multirow[t]{3}{*}{ based on prescription registry $(\mathrm{N}=45607)$} & No & 28821 & 1281 & 1 & 1 & 1 \\
\hline & Yes & 11367 & 627 & 1.24 (1.13 to 1.37$)$ & 1.15 (1.04 to 1.26$)$ & $1.13(1.03$ to 1.25$)$ \\
\hline & Don't know & 4448 & 226 & $1.14(1.00$ to 1.31$)$ & 1.07 (0.93 to 1.23$)$ & $1.07(0.93$ to 1.23$)$ \\
\hline
\end{tabular}

Model 1 adjusted for maternal age, maternal parity, maternal education, maternal salary, maternal pre-pregnancy BMI, and maternal asthma.

Model 2 adjusted for maternal age, maternal parity, maternal education, maternal salary, maternal pre-pregnancy BMI, maternal asthma and maternal smoking during pregnancy.

Multiple imputation of missing covariate information by chained equations conducted for all measures of association presented. There were a total of 1069 children in the analysis of

asthma at 36 months, 535 children in the analysis of asthma at 7 years and 971 children in the analysis of use of asthma medication for whom we imputed missing information on the

grandmother's smoking status. Furthermore, missing information on asthma status was imputed for 610 children with regard to current asthma at 36 months and 540 children with

regard to current asthma at 7 years. For all other covariates, the amount of missing information that was imputed was less than $1.5 \%$. 
the prescription registry. ${ }^{34}$ The prevalence of current asthma at 36 months and 7 years in MoBa was relatively low compared with estimates from the Environment and Childhood Asthma Study in Oslo. ${ }^{35}$ This could be due to the fairly stringent asthma definition used in the current study and/or due to different background characteristics.

A selection bias might have occurred due to the initial participation rate. Comparing $\mathrm{MoBa}$ participants with all pregnant women who gave birth during the $\mathrm{MoBa}$ inclusion period as registered in the birth registry indicated that MoBa participants were older, less likely to be single, less likely to have more than two previous deliveries and less likely to smoke. ${ }^{18}$ However, these differences were not found to influence a number of exposure outcome associations. ${ }^{18}$ Overall, we do not think that the initial participation rate is a strong source of selection bias. However, this could influence the generalisability of our results. The current study further required information from a large number of follow-up questionnaires. We were able to evaluate selection bias to some degree at age 7 by using the prescription registry as an additional outcome. Reassuringly, we saw similar association of the grandmother's smoking with asthma at 7 years in the grandchild classified based on questionnaire data and classified based on the prescription registry (table 2). Furthermore, the analysis using inverse probability weighting to make the study sample more representatives of all eligible $\mathrm{MoBa}$ participants gave similar results.

In contrast to the Children's Health Study, we did not find an independent positive association of maternal smoking during pregnancy with asthma development. ${ }^{9}$ There are some potential explanations for this finding. The Norwegian government banned smoking from all public buildings in 2004. Furthermore, the Norwegian Health Directorate has conducted multiple health awareness campaigns during the MoBa enrolment period targeted at getting pregnant women to stop smoking. The proportion of women who smoke during pregnancy has gone down during the MoBa enrolment period. It is possible that the low proportion of smokers in MoBa compared with earlier studies (13-38\% of mothers smoked during pregnancy in a pooled analysis of eight European birth cohorts) or the moderate smoking among daily smokers (average of seven cigarettes per day) attenuated the power to detect an association between maternal smoking during pregnancy and asthma seen in other studies. ${ }^{2}$

In conclusion, the grandmother's smoking when pregnant with the mother increased the risk of asthma in the grandchild independent of the mother's smoking status. However, given limited information on the grandmother's socioeconomic status, asthma status and other factors, unmeasured confounding may be present.

Acknowledgement We are grateful to all families participating in the Norwegian Mother and Child Cohort Study.

Contributors All authors contributed equally to each phase of the study. WN was responsible for data acquisition. SEH and PN contributed in the design of the work. SJL contributed to interpretation of the data for the work. ØK contributed to the analysis. MCM had the primary responsibility for the data analysis and drafting the paper. All authors contributed to revising the manuscript, approved the final version of the manuscript for submission and contributed to ensuring the accuracy and integrity of the work.

Funding The Norwegian Mother and Child Cohort Study is supported by NIH $(\mathrm{NIH} /$ NIEHS contract number N01-ES-75558, NIH/NINDS grant no. 1 U01 NS 047537-01 and grant no. 2 U01 NS 047537-06A1) and the Norwegian Research Council/FUGE (grant no. 151918/S10). Supported in part by the Intramural Research Program of the NIH, NIEHS (ZIA ES049019). Ms Magnus is also supported by the Norwegian Extra-Foundation for Health and Rehabilitation (grant no. 2011.2.0218).

Competing interests None.

Ethics approval The Regional Ethics Committee for South/East Norway.
Provenance and peer review Not commissioned; externally peer reviewed.

Data sharing statement The raw data underlying the current study will be made available upon request from the Norwegian Mother and Child Steering Committee by contacting Luise-Charlotte Birke (charlotte.birke@fhi.no).

\section{REFERENCES}

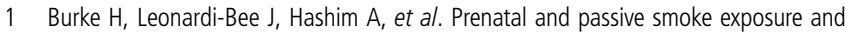
incidence of asthma and wheeze: systematic review and meta-analysis. Pediatrics 2012;129:735-44.

2 Neuman A, Hohmann C, Orsini N, et al. Maternal smoking in pregnancy and asthma in preschool children: a pooled analysis of eight birth cohorts. Am J Respir Crit Care Med 2012;186:1037-43.

3 Vork KL, Broadwin RL, Blaisdell RJ. Developing asthma in childhood from exposure to secondhand tobacco smoke: insights from a meta-regression. Environ Health Perspect 2007;115:1394-400.

4 Gilliland FD, Berhane K, Li YF, et al. Effects of early onset asthma and in utero exposure to maternal smoking on childhood lung function. Am J Respir Crit Care Med 2003;167:917-24.

5 Hayatbakhsh MR, Sadasivam S, Mamun AA, et al. Maternal smoking during and after pregnancy and lung function in early adulthood: a prospective study. Thorax 2009;64:810-14.

6 Li YF, Gilliland FD, Berhane K, et al. Effects of in utero and environmental tobacco smoke exposure on lung function in boys and girls with and without asthma. Am J Respir Crit Care Med 2000;162:2097-104.

7 Moshammer $\mathrm{H}$, Hoek G, Luttmann-Gibson $\mathrm{H}$, et al. Parental smoking and lung function in children: an international study. Am J Respir Crit Care Med 2006;173:1255-63.

8 Rehan VK, Liu J, Sakurai R, et al. Perinatal nicotine-induced transgenerational asthma. Am J Physiol Lung Cell Mol Physiol 2013;305:L501-7.

9 Li YF, Langholz B, Salam MT, et al. Maternal and grandmaternal smoking patterns are associated with early childhood asthma. Chest 2005;127:1232-41.

10 Miller LL, Henderson J, Northstone K, et al. Do grandmaternal smoking patterns influence the aetiology of childhood asthma? Chest 2013;145:1213-8.

11 Miller LL, Pembrey M, Davey SG, et al. Is the growth of the fetus of a non-smoking mother influenced by the smoking of either grandmother while pregnant? PLOS ONE 2014;9:e86781.

12 Misra DP, Astone N, Lynch CD. Maternal smoking and birth weight: interaction with parity and mother's own in utero exposure to smoking. Epidemiology 2005;16:288-93.

13 Rillamas-Sun E, Harlow SD, Randolph JF Jr. Grandmothers' smoking in pregnancy and grandchildren's birth weight: comparisons by grandmother birth cohort. Matern Child Health J 2013;18:1691-8.

14 Bjerg A, Hedman L, Perzanowski $M$, et al. A strong synergism of low birth weight and prenatal smoking on asthma in schoolchildren. Pediatrics 2011;127:e905-e12.

15 Kindlund K, Thomsen SF, Stensballe LG, et al. Birth weight and risk of asthma in 3-9-year-old twins: exploring the fetal origins hypothesis. Thorax 2010;65:146-9.

16 Joubert BR, Haberg SE, Nilsen RM, et al. 450K epigenome-wide scan identifies differential DNA methylation in newborns related to maternal smoking during pregnancy. Environ Health Perspect 2012;120:1425-31.

17 Magnus P, Irgens LM, Haug K, et al. Cohort profile: the Norwegian Mother and Child Cohort Study (MoBa). Int J Epidemiol 2006:35:1146-50.

18 Nilsen RM, Vollset SE, Gjessing HK, et al. Self-selection and bias in a large prospective pregnancy cohort in Norway. Paediatr Perinat Epidemiol 2009;23:597-608.

19 Norwegian Institute of Public Health. The Norwegian Mother and Child Cohort Study Questionnaires. Secondary The Norwegian Mother and Child Cohort Study Questionnaires 2014 (cited 1 Sep 2014). http://www.fhi.no/eway/default.aspx? pid=238\&trg=MainArea_5811\&MainArea_5811=5903:0:15,3138:1:0:0:::0:0.

20 Sheikh K. Investigation of selection bias using inverse probability weighting. Eur $J$ Epidemiol 2007;22:349-50.

21 Patil VK, Holloway JW, Zhang $\mathrm{H}$, et al. Interaction of prenatal maternal smoking, interleukin 13 genetic variants and DNA methylation influencing airflow and airway reactivity. Clin Epigenetics 2013;5:22.

22 Rehan VK, Liu J, Naeem E, et al. Perinatal nicotine exposure induces asthma in second generation offspring. BMC Med 2012;10:129.

23 Joubert BR, Haberg SE, Bell DA, et al. Maternal smoking and DNA methylation in newborns: in utero effect or epigenetic inheritance? Cancer Epidemiol Biomarkers Prev 2014;23:1007-17.

24 Walsh RA. Effects of maternal smoking on adverse pregnancy outcomes: examination of the criteria of causation. Hum Biol 1994;66:1059-92.

25 Wang $X$, Tager IB, Van VH, et al. Maternal smoking during pregnancy, urine cotinine concentrations, and birth outcomes. A prospective cohort study. Int J Epidemiol 1997;26:978-88.

26 Gluckman PD, Hanson MA, Morton SM, et al. Life-long echoes - a critical analysis of the developmental origins of adult disease model. Biol Neonate 2005;87:127-39.

27 Harding JE. The nutritional basis of the fetal origins of adult disease. Int J Epidemiol 2001;30:15-23.

Magnus MC, et al. Thorax 2015;70:237-243. doi:10.1136/thoraxjnl-2014-206438 
28 Haberg SE, Stigum H, London SJ, et al. Maternal obesity in pregnancy and respiratory health in early childhood. Paediatr Perinat Epidemiol 2009;23:352-62.

29 Harpsoe MC, Basit S, Bager P, et al. Maternal obesity, gestational weight gain, and risk of asthma and atopic disease in offspring: a study within the Danish National Birth Cohort. J Allergy Clin Immunol 2013;131:1033-40.

30 Pike KC, Inskip HM, Robinson SM, et al. The relationship between maternal adiposity and infant weight gain, and childhood wheeze and atopy. Thorax 2013:68:372-9.

31 Simard JF, Rosner BA, Michels KB. Exposure to cigarette smoke in utero: comparison of reports from mother and daughter. Epidemiology 2008;19:628-33.
32 Cupul-Uicab LA, Ye X, Skjaerven $\mathrm{R}$, et al. Reproducibility of reported in utero exposure to tobacco smoke. Ann Epidemiol 2011;21:48-52.

33 Kvalvik LG, Nilsen RM, Skjaerven R, et al. Self-reported smoking status and plasma cotinine concentrations among pregnant women in the Norwegian Mother and Child Cohort Study. Pediatr Res 2012;72:101-7.

34 Furu K, Karlstad 0, Skurtveit S, et al. High validity of mother-reported use of antiasthmatics among children: a comparison with a population-based prescription database. J Clin Epidemiol 2011;64:878-84.

35 Lodrup Carlsen KC, Haland G, Devulapalli CS, et al. Asthma in every fifth child in Oslo, Norway: a 10-year follow up of a birth cohort study. Allergy 2006;61:454-60. 